\title{
Studies on Performance of White Onion Advance Lines for Growth and Yield for Selection of Quality Variety
}

\author{
R. K. Singh ${ }^{1 *}$, S. K. Singh ${ }^{2}$ and A. K. Tailor ${ }^{3}$ \\ ${ }^{1}$ Department of Vegetable Science, Banda University of Agriculture and Technology, \\ Banda UP, India \\ ${ }^{2} \mathrm{NHRDF}$, New Delhi, India \\ ${ }^{3}$ National Horticultural Research and Development Foundation, \\ Nashik, Maharashtra, India \\ *Corresponding author
}

\section{A B S T R A C T}

\begin{tabular}{|l|}
\hline Key w o r d s \\
Onion, Allium cepa, \\
Genotypes, \\
Evaluation, \\
Utilization of onion \\
\hline Article Info \\
\hline $\begin{array}{l}\text { Accepted: } \\
\text { 26 May } 2020 \\
\text { Available Online: } \\
\text { 10 June } 2020\end{array}$ \\
\hline
\end{tabular}

India is second largest producer of onion in the world after China. Onion (Allium cepa L.) is commercially cultivated and widely consumed as vegetable and as spices in India. About 73.23 million tons of onions are produced in the world from 3.65 million ha area. India, being major onion-producing country, produces 20.13 million tons from 1.19 million ha, with a very low productivity of $16.24 \mathrm{t} / \mathrm{ha}$ in comparison to Republic of Korea (64.58 t/ha), USA (54.47 t/ha), Spain (53.69 t/ha), Netherland (45.80 t/ha), Japan (42.46 t/ha), Germany (41.86 t/ha) and United Kingdom (41.15 t/ha). Lack of recommended or released variety of high yielding as well as good keeping quality in the country, it creates price fluctuation during off season arrival period. To meet out the domestic requirement and also to fulfill the export demand, selection of high yielding genotype under different agro-climatic conditions is necessary. The experiment was conducted at Nashik and Karnal during Rabi 2017-18. The trial conducted at Nashik, revealed that the highest gross yield $(382.13 \mathrm{q} / \mathrm{ha})$ and marketable yield $(250.47$ q/ha) were recorded in advance line-874 which was at par with line-827 in respect of marketable yield. Highest TSS $(13.05 \%)$ and dry matter content $(14.44 \%)$ were noted in line-830 and found at par with lines-562, 799, 823, 832, 865, 886 and Agrifound White, however at Karnal, the highest gross yield (290.28 q/ha) and marketable yield $(258.82 \mathrm{q} / \mathrm{ha}$ ) were recorded in lines-810 and 562, respectively, which were par with advance line-562 in respect of gross yield. The highest total soluble solids $(14.40 \%)$ were recorded in advance line- 810 . It is concluded that the above genotypes can be utilized for white onion breeding programme for develop a good quality variety for different agroclimatic condition.

\section{Introduction}

Onion is one of the most important underground bulbous vegetable crops grown in India, having both the food and medicinal values. It is widely cultivated for internal consumption as well as for the export. India is the second largest producer of onion in the world after China. About 73.23 million tons of onions are produced in the world from 3.65 million ha area. India, being major onionproducing country, produces 20.13 million tons from 1.19 million ha, with a very low productivity of $16.24 \mathrm{t} / \mathrm{ha}$ in comparison to Republic of Korea (64.58 t/ha), USA (54.47 t/ha), Spain (53.69 t/ha), Netherland (45.80 t/ha), Japan (42.46 t/ha), Germany (41.86 t/ha) and United Kingdom (41.15 t/ha). The white onion with high total soluble solids highly utilized for dehydration purpose such 
as flakes powder, paste, crush and pickle, etc. (Singh et al., 2004).Processed onion, highly competitive commodity in the international market should possess certain desirable traits such as high yield retentive attractive colour, high pungency and good drying ratio (Kurade and Mathias, 1972).The main white onion growing states in India are Maharashtra, Gujarat, Karnataka and Madhya Pradesh. and has many medicinal properties. The production of white onion is now becoming popular among farmers, producers and exporter. The exporters export the white onion from Maharashtra and Gujarat and they are demanding a good white onion variety which has greater potential for dehydration.

About $55-60 \%$ of onion comes from Rabi season and 40-45\% from Kharif and late Kharif season. Because of its high export potential, it comes under cash crop apart from vegetable (Pandey, 1989). It is predominantly a Rabi season crop and most onion cultivars are sensitive to photo period and thus their range of adoption is limited (Gupta and Singh, 2010). Lack of recommended or released variety of high yielding as well as good keeping quality in the country, it creates price fluctuation during off season arrival period.

The work conducted on selection of suitable white onion is very scanty (Saimbhi et al., 1971 and Sethi et al., 1993). To meet out the domestic requirement and also to fulfill the export demand, selection of high yielding genotype under different agro- climatic conditions is necessary. In onion, local genotypes play important role in development of new cultivars. The main onion growing states in India are Maharashtra, Gujarat, Karnataka, Tamil Nadu, Odisha, Madhya Pradesh, Uttar Pradesh, Andhra Pradesh, Bihar and Punjab. It is used as a salad or cooked in various ways in all curies, fried or baked and also used in processed form e.g. flakes powder, paste, crush and pickle, etc. National Horticultural Research and Development Foundation, Nashik, collected good number of germplasm and evaluated their performance regarding different attributes. Hence under present study a total of 17 germplasm along with one check Agrifound White at Nashik while at Karnal also 14 genotypes along with one check Agrifound White were evaluated to assess their performance for selection of high yielding varieties for Rabi season.

\section{Materials and Methods}

The present investigation was carried out at National Horticultural Research and Development Foundation at Nashik, Maharashtra and Karnal, Haryana during Rabi 2017-18. The experiment was laid out in randomized block design with three replications. The Nashik $\left(20^{\circ} \mathrm{N}\right.$ latitude and $73^{0} \mathrm{E}$ longitudes) is located at altitude of 492 meter above mean sea levels. The minimum and maximum temperature and humidity is ranging between $10{ }^{0} \mathrm{C}$ to $40{ }^{0} \mathrm{C}$ and $48 \%$ to $80 \%$, respectively, with an annual rain fall around $881 \mathrm{~mm}$.

The soil of the trial was clay loam, medium in organic carbon $(0.58 \%)$, available nitrogen (385.2 kg/ha), phosphorus (45.13kg/ha) and high in available potash $(291.2 \mathrm{~kg} / \mathrm{ha})$. The study comprises under present study a total of 17 germplasm along with one check Agrifound White at Nashik, while at Karnal also 14 genotypes along with one check Agrifound White selected among more than 200 genotypes evaluated at this centre. The seeds were sown on 4/11/2017 and seedlings were transplanted on 09/01/2018 at Nashik, while at Karnal, the seeds were sown on $13 / 11 / 2017$ and seedlings were transplanted on $28 / 01 / 2018$, however, harvesting was done as per maturity of bulbs at Nashik from $24 / 04 / 2018$ to $28 / 04 / 2018$ and at Karnal on 
04/05/2018-14/05/2018. 50 to 55 days old seedlings of each onion genotypes were transplanted in flat beds in the spacing of 15 $\mathrm{cm} \times 10 \mathrm{~cm}$ in a plot of $3.6 \mathrm{~m}$ x $1.8 \mathrm{~m}$ size. The recommended package of practices was uniformly followed during whole experiment period to raise a successful crop. Randomly selected ten plants from each plot were taken to record the observations on plant establishment $(\%)$, plant height $(\mathrm{cm})$, leaves per plant, neck thickness $(\mathrm{cm})$, equatorial bulb diameter $(\mathrm{cm})$, polar bulb diameter, P: E ratio, weight of 20 bulbs $(\mathrm{kg})$, days for harvesting, doubles $(\%)$, bolters $(\%)$, rotten $\%$, total soluble solid (\%), dry matter content $(\%)$, gross yield (q/ha), marketable yield (q/ha), skin intactness at harvest, compactness of bulbs, colour of bulbs, shape of bulbs, thrips incidence and intensity, stemphylium blight intensity. The data were analyzed to find out the superior genotypes for development of good quality onion varieties suitable for different agro climatic conditions.

\section{Results and Discussion}

At Nashik, the data presented in Table-1 observed that traits such as number of leaves per plant, neck thickness, equatorial bulb diameter, polar bulb diameter and P: E ratio, exhibited non-significant differences. Highest $\%$ of plant establishment (96.97) and plant height $(69.53 \mathrm{~cm})$ were noted in line-832 and check variety Agrifound White, respectively, and were at par with lines-784, 823 and 885 in respect of \% of plant establishment and lines-799, 869 and 857 regarding plant height.

Highest 20 bulbs weight (1.16 kg) was recorded in line-830. The significant and highest bulb diameter and bulb weight was recorded in white onion variety Agrifound White and others advance lines (Singh et al., 2010; Singh, 1991; Mohanty, 2001; Patel et al., 1985; Sidhu et al., 1986; Singh et al., 2010; Singh et al., 2010; Singh et al., 2011 and Singh et al., 2011) reported that bulb diameter; size index and weight of bulb had correlated positively and increases the total yield. Lowest doubles on number basis $(0.29$ $\%)$ were recorded in advance line-886 and it was at par with advance lines-562, 799, 810, $823,827,869,885$ and 857.

No bolters on number basis was recorded in advance line-830, however, no rotten bulbs were recorded in any line except line-562, 784 and 830. The advance lines which showed minimum bolters and doubles can be utilized for good quality onion bulb variety. (Bhonde et al., 1991) also recorded similar range of bolting in their study of different varieties.

The highest TSS (13.05\%) and dry matter (14.44\%) were recorded in advance line-830 and was at par with advance lines-562, 799, 823, 832, 865, 886 and Agrifound White. Highest gross yield (382.13 q/ha) and marketable yield (250.47 q/ha) were recorded in line-874 and was at par with line-827 in respect of marketable yield. Minimum duration (105 days) for maturity was taken by lines-793, 799 and 886. The duration for maturity ranged from 105 to 109 days.

The data of Karnal presented in Table- 2 revealed that the trait $\mathrm{P}: \mathrm{E}$ ratio exhibited nonsignificant differences. The highest $\%$ of plant establishment (98.61) was recorded in advance line-810 and it was at par with all the advance lines except 784, 823, 827 and 874 . Maximum plant height $(69.07 \mathrm{~cm})$ and no of leaves per plant (7.13) were recorded in advance line-793 and 830, respectively and was at par with lines-562, 810, 827, 830, 836 and 869 in respect of plant height, while lines$562,784,793,823$ and 869 regarding number of leaves per plant. Thinnest neck $(1.53 \mathrm{~cm})$ was recorded in lines-832, 874, 885 and Agrifound White which was at par with lines784, 827, 830, and 857. 
Table.1 Performance of white onion advance line at Nashik during Rabi, 2017-18

\begin{tabular}{|l|c|c|c|c|c|c|c|c|}
\hline Advance lines & $\begin{array}{c}\text { Plant } \\
\text { Establish- } \\
\text { ment } \mathbf{( \% )}\end{array}$ & $\begin{array}{c}\text { Plant } \\
\text { height } \\
\text { (cm) }\end{array}$ & $\begin{array}{c}\text { No. of } \\
\text { leaves/ } \\
\text { plant }\end{array}$ & $\begin{array}{c}\text { Neck } \\
\text { thickness } \\
\text { (cm) }\end{array}$ & $\begin{array}{c}\text { Equatorial } \\
\text { bulb } \\
\text { diameter }(\mathbf{c m})\end{array}$ & $\begin{array}{c}\text { Polar } \\
\text { bulb } \\
\text { diameter }\end{array}$ & $\begin{array}{c}\text { P:Em) } \\
\text { Ratio }\end{array}$ & $\begin{array}{c}\text { Weight of } \\
\mathbf{2 0} \text { bulbs } \\
\text { (kg) }\end{array}$ \\
\hline L-562 & 82.41 & 64.40 & 8.80 & 1.43 & 5.43 & 3.61 & 1.51 & 1.09 \\
\hline L-784 & 85.12 & 65.80 & 8.33 & 1.41 & 5.08 & 3.70 & 1.37 & 1.04 \\
\hline L-793 & 72.22 & 66.60 & 8.33 & 1.41 & 5.30 & 3.61 & 1.47 & 1.04 \\
\hline L-799 & 73.61 & 66.93 & 8.67 & 1.39 & 5.23 & 3.68 & 1.42 & 0.95 \\
\hline L-810 & 62.73 & 65.67 & 8.40 & 1.37 & 5.29 & 3.51 & 1.51 & 0.92 \\
\hline L-823 & 87.33 & 66.20 & 8.53 & 1.38 & 5.35 & 3.52 & 1.52 & 1.03 \\
\hline L-827 & 79.17 & 61.67 & 8.53 & 1.40 & 5.45 & 3.57 & 1.53 & 1.01 \\
\hline L-830 & 84.38 & 65.80 & 8.60 & 1.40 & 4.30 & 3.46 & 1.24 & 1.16 \\
\hline L-832 & 96.97 & 64.47 & 8.47 & 1.39 & 5.31 & 3.38 & 1.57 & 0.98 \\
\hline L-836 & 78.70 & 64.23 & 8.80 & 1.40 & 5.19 & 3.41 & 1.52 & 0.92 \\
\hline L-842 & 71.15 & 65.73 & 8.53 & 1.36 & 5.23 & 3.47 & 1.51 & 0.94 \\
\hline L-865 & 77.31 & 64.87 & 8.40 & 1.43 & 5.36 & 3.54 & 1.52 & 0.93 \\
\hline L-869 & 75.40 & 67.87 & 8.20 & 1.42 & 5.39 & 3.43 & 1.57 & 0.96 \\
\hline L-874 & 82.29 & 65.00 & 8.53 & 1.37 & 5.29 & 3.63 & 1.46 & 0.99 \\
\hline L-885 & 85.37 & 65.73 & 8.40 & 1.42 & 5.33 & 3.63 & 1.47 & 0.98 \\
\hline L-886 & 78.47 & 65.27 & 9.00 & 1.37 & 5.40 & 3.53 & 1.53 & 0.99 \\
\hline L-857 & 76.92 & 69.20 & 8.67 & 1.42 & 5.29 & 3.51 & 1.51 & 0.94 \\
\hline Agrifound white $(\mathbf{C})$ & 78.65 & 69.53 & 8.93 & 1.41 & 5.31 & 3.53 & 1.50 & 0.99 \\
\hline S Em \pm & $\mathbf{6 . 1 0}$ & $\mathbf{1 . 3 7}$ & $\mathbf{0 . 3 1}$ & $\mathbf{0 . 0 5}$ & $\mathbf{0 . 3 5}$ & $\mathbf{0 . 1 1}$ & $\mathbf{0 . 1 0}$ & $\mathbf{0 . 0 4}$ \\
\hline CD at 5\% & $\mathbf{1 2 . 4 0}$ & $\mathbf{2 . 7 8}$ & $\mathbf{N S}$ & $\mathbf{N S}$ & $\mathbf{N S}$ & $\mathbf{N S}$ & $\mathbf{N S}$ & $\mathbf{0 . 0 8}$ \\
\hline CV \% & $\mathbf{9 . 4 1}$ & $\mathbf{2 . 5 5}$ & $\mathbf{4 . 3 7}$ & $\mathbf{3 . 9 9}$ & $\mathbf{8 . 1 1}$ & $\mathbf{3 . 8 2}$ & $\mathbf{8 . 5 2}$ & $\mathbf{5 . 4 0}$ \\
\hline
\end{tabular}

\begin{tabular}{|c|c|c|c|c|c|c|c|c|c|}
\hline \multirow{2}{*}{$\begin{array}{l}\text { Advance lines } \\
\text { L-562 }\end{array}$} & \multicolumn{2}{|c|}{$\begin{array}{c}\text { Bolters on } \\
\text { number basis } \\
(\%)\end{array}$} & \multicolumn{2}{|c|}{$\begin{array}{c}\text { Doubles on } \\
\text { number basis } \\
(\%)\end{array}$} & \multicolumn{2}{|c|}{$\begin{array}{c}\text { Rotten } \\
(\%)\end{array}$} & \multirow{2}{*}{$\begin{array}{l}\text { TSS } \\
(\%) \\
12.82 \\
\end{array}$} & \multirow{2}{*}{$\begin{array}{c}\begin{array}{c}\text { Dry } \\
\text { matter } \\
(\%)\end{array} \\
14.11\end{array}$} & \multirow{2}{*}{$\begin{array}{r}\begin{array}{c}\text { Gross yield } \\
\text { (q/ha) }\end{array} \\
278.87\end{array}$} \\
\hline & 1.69 & $(1.29)$ & 0.55 & (1.10) & 0.55 & $(0.97)$ & & & \\
\hline L-784 & 1.16 & (1.12) & 0.71 & $(1.25)$ & 0.44 & $(0.71)$ & 12.20 & 13.51 & 217.84 \\
\hline L-793 & 0.76 & (1.68) & 1.06 & $(1.37)$ & 0.00 & $(0.71)$ & 11.51 & 12.88 & 208.57 \\
\hline L-799 & 2.37 & (1.51) & 1.39 & (1.10) & 0.00 & $(0.71)$ & 12.96 & 14.23 & 292.16 \\
\hline L-810 & 1.80 & (1.28) & 0.71 & (1.05) & 0.00 & $(0.71)$ & 12.13 & 13.49 & 313.98 \\
\hline L-823 & 1.18 & $(1.28)$ & 0.60 & (1.05) & 0.00 & $(0.71)$ & 12.89 & 14.25 & 197.51 \\
\hline L-827 & 2.19 & (1.63) & 0.41 & $(0.95)$ & 0.00 & $(0.71)$ & 12.02 & 13.38 & 298.73 \\
\hline L-830 & 0.00 & $(0.71)$ & 0.82 & (1.14) & 0.60 & $(1.05)$ & 13.05 & 14.44 & 232.08 \\
\hline L-832 & 0.93 & $(1.20)$ & 1.20 & $(1.30)$ & 0.00 & $(0.71)$ & 12.98 & 14.39 & 315.26 \\
\hline L-836 & 1.75 & (1.50) & 1.17 & (1.28) & 0.00 & $(0.71)$ & 12.58 & 14.02 & 244.33 \\
\hline L-842 & 1.50 & $(1.41)$ & 0.89 & (1.18) & 0.00 & $(0.71)$ & 11.16 & 12.56 & 244.69 \\
\hline L-865 & 0.59 & (1.04) & 0.91 & (1.19) & 0.00 & $(0.71)$ & 12.67 & 14.07 & 245.86 \\
\hline L-869 & 0.76 & (1.12) & 0.52 & (1.01) & 0.00 & $(0.71)$ & 12.31 & 13.71 & 249.00 \\
\hline L-874 & 2.12 & (1.61) & 1.68 & (1.46) & 0.00 & $(0.71)$ & 11.64 & 13.03 & 382.13 \\
\hline L-885 & 0.60 & $(1.05)$ & 0.39 & $(0.93)$ & 0.00 & $(0.71)$ & 10.67 & 12.17 & 203.72 \\
\hline L-886 & 0.87 & (1.13) & 0.29 & $(0.86)$ & 0.00 & $(0.71)$ & 12.93 & 14.25 & 260.71 \\
\hline L-857 & 0.80 & (1.10) & 0.57 & (1.01) & 0.00 & $(0.71)$ & 11.27 & 12.67 & 288.84 \\
\hline Agrifound white (C) & 0.88 & (1.17) & 1.10 & $(1.26)$ & 0.00 & $(0.71)$ & 12.78 & 14.07 & 244.37 \\
\hline S Em \pm & - & 0.14 & - & 0.12 & - & 0.01 & 0.21 & 0.20 & 20.55 \\
\hline CD at $5 \%$ & - & 0.28 & - & 0.24 & - & 0.02 & 0.43 & 0.41 & 41.76 \\
\hline CV \% & - & 13.35 & - & 12.85 & - & 1.42 & 2.10 & 1.78 & 9.60 \\
\hline
\end{tabular}

Note: Data shows in parenthesis Square root transformed value 


\begin{tabular}{|l|c|c|c|c|c|c|}
\hline \multicolumn{1}{|c|}{ Advance lines } & $\begin{array}{c}\text { Marketable } \\
\text { yield } \\
\text { (q/ha) }\end{array}$ & $\begin{array}{c}\text { Days for } \\
\text { maturity }\end{array}$ & $\begin{array}{c}\text { Skin } \\
\text { intactness }\end{array}$ & $\begin{array}{c}\text { Compactness } \\
\text { of bulb }\end{array}$ & $\begin{array}{c}\text { Color } \\
\text { of bulb }\end{array}$ & $\begin{array}{c}\text { Shape of } \\
\text { bulb }\end{array}$ \\
\hline L-562 & 193.31 & 108 & Tight & Compact & White & G Round \\
\hline L-784 & 132.06 & 107 & Tight & Compact & White & G Round \\
\hline L-793 & 176.21 & 105 & Tight & Compact & White & G Round \\
\hline L-799 & 189.49 & 105 & Tight & Compact & White & G Round \\
\hline L-810 & 190.37 & 109 & Tight & Compact & White & G Round \\
\hline L-823 & 115.79 & 108 & Tight & Compact & White & G Round \\
\hline L-827 & 229.76 & 108 & Tight & Compact & White & G Round \\
\hline L-830 & 140.88 & 109 & Tight & Compact & White & G Round \\
\hline L-832 & 189.60 & 109 & Tight & Compact & White & G Round \\
\hline L-836 & 163.54 & 107 & Tight & Compact & White & G Round \\
\hline L-842 & 162.15 & 109 & Tight & Compact & White & G Round \\
\hline L-865 & 157.88 & 109 & Tight & Compact & White & G Round \\
\hline L-869 & 156.34 & 106 & Tight & Compact & White & G Round \\
\hline L-874 & 250.47 & 108 & Tight & Compact & White & G Round \\
\hline L-885 & 160.91 & 109 & Tight & Compact & White & G Round \\
\hline L-886 & 188.21 & 105 & Tight & Compact & White & G Round \\
\hline L-857 & 218.58 & 109 & Tight & Compact & White & G Round \\
\hline Agrifound white (C) & 185.52 & 109 & Tight & Compact & White & G Round \\
\hline S Em \pm & $\mathbf{1 4 . 4 7}$ & - & - & - & - & - \\
\hline CD at 5\% & $\mathbf{2 9 . 4 1}$ & - & - & - & - & - \\
\hline CV \% & $\mathbf{9 . 9 7}$ & - & - & - & - & - \\
\hline & & & & - & - & - \\
\hline
\end{tabular}

Table.2 Performance of white onion advance lines at Karnal during Rabi, 2017-18

\begin{tabular}{|l|c|c|c|c|c|c|c|c|}
\hline Advance lines & $\begin{array}{c}\text { Plant } \\
\text { Establish- } \\
\text { ment } \mathbf{( \% )}\end{array}$ & $\begin{array}{c}\text { Plant } \\
\text { height } \\
\text { (cm) }\end{array}$ & $\begin{array}{c}\text { No. of } \\
\text { leaves/ } \\
\text { plant }\end{array}$ & $\begin{array}{c}\text { Neck } \\
\text { thickness } \\
\text { (cm) }\end{array}$ & $\begin{array}{c}\text { Equatorial } \\
\text { bulb } \\
\text { diameter } \\
(\mathbf{c m})\end{array}$ & $\begin{array}{c}\text { Polar } \\
\text { bulb } \\
\text { diameter }(\mathbf{c m})\end{array}$ & $\begin{array}{c}\text { P:E } \\
\text { Ratio }\end{array}$ & $\begin{array}{c}\text { Weight of 20 } \\
\text { Bulbs } \\
\text { (kg) }\end{array}$ \\
\hline L-562 & 97.92 & 68.27 & 6.93 & 1.64 & 5.01 & 3.77 & 1.33 & 1.16 \\
\hline L-784 & 94.44 & 66.00 & 6.93 & 1.58 & 5.15 & 3.54 & 1.46 & 1.15 \\
\hline L-793 & 95.00 & 69.07 & 6.93 & 1.62 & 4.94 & 3.51 & 1.41 & 1.17 \\
\hline L-810 & 98.61 & 68.93 & 7.07 & 1.73 & 5.00 & 3.73 & 1.34 & 1.21 \\
\hline L-823 & 93.33 & 67.60 & 6.93 & 1.61 & 5.04 & 3.59 & 1.40 & 1.07 \\
\hline L-827 & 94.72 & 68.93 & 6.60 & 1.59 & 4.89 & 3.59 & 1.37 & 1.16 \\
\hline L-830 & 96.39 & 68.67 & 7.13 & 1.58 & 4.84 & 3.46 & 1.40 & 1.09 \\
\hline L-832 & 97.50 & 60.07 & 6.13 & 1.53 & 4.79 & 3.63 & 1.32 & 1.02 \\
\hline L-836 & 97.78 & 68.40 & 6.67 & 1.58 & 4.88 & 3.61 & 1.35 & 1.04 \\
\hline L-865 & 97.78 & 66.67 & 6.33 & 1.60 & 4.83 & 3.53 & 1.37 & 1.13 \\
\hline L-869 & 96.39 & 68.27 & 7.00 & 1.60 & 4.78 & 3.43 & 1.40 & 1.18 \\
\hline L-874 & 93.89 & 66.27 & 6.67 & 1.53 & 4.97 & 3.45 & 1.44 & 1.09 \\
\hline L-885 & 97.22 & 63.60 & 6.13 & 1.53 & 4.61 & 3.25 & 1.42 & 1.01 \\
\hline L-857 & 97.22 & 63.27 & 6.07 & 1.57 & 4.88 & 3.53 & 1.38 & 1.04 \\
\hline Agrifound white (C) & 95.83 & 67.53 & 6.33 & 1.53 & 4.55 & 3.42 & 1.33 & 0.81 \\
\hline S Em \pm & $\mathbf{1 . 3 0}$ & $\mathbf{0 . 6 8}$ & $\mathbf{0 . 1 1}$ & $\mathbf{0 . 0 3}$ & $\mathbf{0 . 1 4}$ & $\mathbf{0 . 1 2}$ & $\mathbf{0 . 0 6}$ & $\mathbf{0 . 0 5}$ \\
\hline CD at 5\% & $\mathbf{2 . 6 6}$ & $\mathbf{1 . 3 9}$ & $\mathbf{0 . 2 3}$ & $\mathbf{0 . 0 6}$ & $\mathbf{0 . 2 9}$ & $\mathbf{0 . 2 5}$ & NS & $\mathbf{0 . 1 0}$ \\
\hline CV \% & $\mathbf{1 . 6 6}$ & $\mathbf{1 . 2 5}$ & $\mathbf{2 . 0 8}$ & $\mathbf{2 . 1 4}$ & $\mathbf{3 . 4 2}$ & $\mathbf{4 . 3 0}$ & $\mathbf{5 . 1 9}$ & $\mathbf{6 . 0 5}$ \\
\hline
\end{tabular}




\begin{tabular}{|c|c|c|c|c|c|c|c|c|c|c|c|c|}
\hline \multirow{2}{*}{$\begin{array}{l}\text { Advance lines } \\
\text { L-562 }\end{array}$} & \multicolumn{2}{|c|}{$\begin{array}{c}\text { Bolters on } \\
\text { number } \\
\text { basis }(\%)\end{array}$} & \multicolumn{2}{|c|}{$\begin{array}{c}\text { Doubles on } \\
\text { number } \\
\text { basis }(\%)\end{array}$} & \multicolumn{2}{|c|}{$\begin{array}{c}\text { Rotten } \\
(\%)\end{array}$} & \multirow{2}{*}{$\begin{array}{l}\text { TSS } \\
(\%)\end{array}$} & \multirow{2}{*}{$\begin{array}{l}\begin{array}{l}\text { Gross } \\
\text { Yield } \\
(\mathbf{q} / \mathbf{h a})\end{array} \\
287.43 \\
\end{array}$} & \multirow{2}{*}{$\begin{array}{c}\text { Market- } \\
\text { able } \\
\text { yield } \\
\text { (q/ha) } \\
258.82\end{array}$} & \multicolumn{2}{|c|}{$\begin{array}{c}\text { Stemphylium } \\
\text { blight } \\
\text { Int. (\%) }\end{array}$} & \multirow{2}{*}{$\begin{array}{r}\text { Thrips } \\
\text { per } \\
\text { plant }\end{array}$} \\
\hline & 0.00 & $(0.71)$ & 1.06 & $(1.25)$ & 2.12 & $(1.62)$ & & & & 17.50 & $(4.24)$ & \\
\hline L-784 & 0.00 & $(0.71)$ & 2.36 & (1.68) & 3.23 & (1.93) & 12.60 & 258.11 & 214.11 & 19.33 & $(4.45)$ & 34.93 \\
\hline L-793 & 0.00 & $(0.71)$ & 3.22 & (1.92) & 8.48 & $(3.00)$ & 13.80 & 262.22 & 207.78 & 16.67 & $(4.13)$ & 42.93 \\
\hline L-810 & 0.00 & $(0.71)$ & 3.95 & (2.09) & 8.46 & (2.99) & 14.40 & 290.28 & 216.02 & 12.60 & $(3.62)$ & 43.20 \\
\hline L-823 & 0.00 & $(0.71)$ & 4.46 & $(2.23)$ & 17.55 & $(4.24)$ & 12.47 & 252.33 & 119.56 & 21.80 & $(4.72)$ & 43.20 \\
\hline L-827 & 0.00 & $(0.71)$ & 1.50 & (1.41) & 4.98 & (2.34) & 12.50 & 258.43 & 192.69 & 17.77 & $(4.27)$ & 38.40 \\
\hline L-830 & 0.29 & $(0.86)$ & 0.56 & (1.03) & 2.29 & (1.66) & 11.67 & 261.48 & 217.09 & 15.03 & (3.94) & 39.73 \\
\hline L-832 & 0.00 & $(0.71)$ & 6.54 & (2.65) & 13.33 & (3.71) & 13.07 & 227.17 & 168.46 & 20.43 & $(4.57)$ & 43.73 \\
\hline L-836 & 0.00 & $(0.71)$ & 3.69 & (2.04) & 2.26 & (1.55) & 12.57 & 239.50 & 190.59 & 14.53 & $(3.87)$ & 42.40 \\
\hline L-865 & 0.00 & $(0.71)$ & 0.00 & $(0.71)$ & 3.74 & $(2.05)$ & 12.47 & 237.87 & 194.63 & 13.60 & $(3.75)$ & 43.73 \\
\hline L-869 & 0.00 & $(0.71)$ & 3.75 & (2.06) & 4.32 & (2.19) & 12.67 & 261.48 & 191.67 & 13.33 & $(3.72)$ & 42.40 \\
\hline L-874 & 0.88 & (1.17) & 1.78 & (1.51) & 6.51 & (2.65) & 12.07 & 214.33 & 176.30 & 17.47 & $(4.23)$ & 43.47 \\
\hline L-885 & 0.00 & $(0.71)$ & 3.44 & (1.98) & 3.43 & (1.98) & 12.27 & 224.07 & 196.11 & 14.83 & $(3.90)$ & 40.27 \\
\hline L-857 & 0.29 & $(0.86)$ & 5.17 & $(2.38)$ & 3.12 & (1.90) & 11.33 & 235.30 & 205.00 & 16.03 & $(4.06)$ & 42.40 \\
\hline $\begin{array}{l}\text { Agrifound } \\
\text { white (C) }\end{array}$ & 0.48 & $(0.98)$ & 4.35 & (2.19) & 7.27 & (2.78) & 12.47 & 215.31 & 176.42 & 17.13 & $(4.20)$ & 38.93 \\
\hline S Em \pm & - & 0.09 & - & 0.15 & - & 0.2 & 0.25 & 12.32 & 14.15 & - & 0.18 & 1.96 \\
\hline CD at $5 \%$ & - & 0.18 & - & 0.31 & - & 0.41 & 0.51 & 25.24 & 28.98 & - & 0.37 & 4.01 \\
\hline CV \% & - & 14.96 & - & 9.9 & - & 9.94 & 2.40 & 6.08 & 8.88 & - & 5.46 & 5.89 \\
\hline
\end{tabular}

Note: Data shows in parenthesis Square root transformed value

\begin{tabular}{|l|c|c|c|c|c|c|}
\hline \multicolumn{1}{|c|}{ Advance lines } & $\begin{array}{c}\text { Days for } \\
\text { maturity }\end{array}$ & $\begin{array}{c}\text { Thrips Inc. } \\
(\mathbf{\%})\end{array}$ & Skin intactness & $\begin{array}{c}\text { Compactness } \\
\text { of bulb }\end{array}$ & $\begin{array}{c}\text { Color } \\
\text { of bulb }\end{array}$ & $\begin{array}{c}\text { Shape } \\
\text { of bulb }\end{array}$ \\
\hline L-562 & 107 & 100 & Tight & Compact & White & Round \\
\hline L-784 & 107 & 100 & Tight & Compact & White & Round \\
\hline L-793 & 104 & 100 & Tight & Compact & White & Round \\
\hline L-810 & 104 & 100 & Tight & Compact & White & Round \\
\hline L-823 & 107 & 100 & Tight & Compact & White & Round \\
\hline L-827 & 104 & 100 & Tight & Compact & White & Round \\
\hline L-830 & 107 & 100 & Tight & Compact & White & Round \\
\hline L-832 & 97 & 100 & Tight & Compact & White & Round \\
\hline L-836 & 104 & 100 & Tight & Compact & White & Round \\
\hline L-865 & 104 & 100 & Tight & Compact & White & Round \\
\hline L-869 & 107 & 100 & Tight & Compact & White & Round \\
\hline L-874 & 107 & 100 & Tight & Compact & White & Round \\
\hline L-885 & 107 & 100 & Tight & Compact & White & Round \\
\hline L-857 & 100 & 100 & Tight & Compact & White & Round \\
\hline Agrifound white (C) & 104 & 100 & Tight & Compact & White & Round \\
\hline S Em \pm & - & - & - & - & - & - \\
\hline CD at 5\% & - & - & - & - & - & - \\
\hline CV \% & - & - & - & - & - & - \\
\hline
\end{tabular}


The highest equatorial bulb diameter (5.15 $\mathrm{cm})$ and polar bulb diameter $(3.77 \mathrm{~cm})$ were recorded in advance lines-784 and 562, respectively and found at par with all the lines except, lines-830, 832, 865, 869, 885 and variety Agrifound White in respect equatorial bulb diameter, while lines-793, 830, 869, 874, 885 and Agrifound White regarding polar bulb diameter.

The highest weight of 20 bulbs $(1.21 \mathrm{~kg})$ was recorded in line-810 and it was at par with lines-562, 784, 793, 827, 865 and 869. Lowest rotten bulbs on number basis $(2.26 \%)$ were recorded in line-836 and it was at par with lines-562, 784, 830 and 857. No doubles were recorded in advance line-865. However, no bolters were recorded in any lines except advance lines-830, 857 and Agrifound White.

The highest total soluble solids $(14.40 \%)$ were recorded in advance line-810. Pungency is one of the important traits which considered as per the consumers preferences. It is suggested that the line which have high total soluble solid and dry matter content can be used for dehydration purpose. Similar findings were also reported by (Verma et al., 1999).

Highest gross yield (290.28 q/ha) and marketable yield ( $258.82 \mathrm{q} / \mathrm{ha})$ were recorded in advance lines-810 and 562, respectively and were found at par with line-562 in respect of gross yield. $100 \%$ stemphylium blight and thrips incidence were recorded in all the lines, however, the lowest intensity of stemphylium blight $(12.60 \%)$ and thrips population (32.00 nymphs/plant) were recorded in advance lines-810 and 562, respectively and was at par with lines-830, 836, 865, 869, and 885 in respect of stemphylium blight intensity and line-784 regarding thrips population. Minimum duration (97 days) for maturity was taken by advance line-832. The duration for maturity ranged from 97 to 107 days.

\section{References}

Bhonde, S. R., Shrivastava, K. J. and Singh, K. N. 1991. Evaluation of varieties for late Kharif (Rangda) crop of onion in Nashik area. Associated Agricultural Development Foundation News letter, XII (I): 1-2

Gupta, R. P and Singh, R. K. 2010. Onion Production in India. Published by Director, National Horticultural Research and Development Foundation Chitegaon Phata, Post-Darna Sangavi, Taluka-Niphad, Dist- Nashik, Maharashtra. Malhotra Publishing house, B-6, DSIDC Complex, Kirti Nagar, New Delhi. 1-88pp

Kurade, A.G. and M. Mathias 1972. Plan for promotion of export of dehydrated onion from India. Ind. Food Packer 26(8): 17

Mohanty, B. K. 2001. Genetic variability, inter relationship and path analysis in onion. J. Tropical Agriculture,39:17-20.

Pandey, U. B. 1989. Onion (Allium cepa L.) Indian Horticulture,33-34:58-62

Patel, R. P., Prasad, M. and Sharma, R. P. 1985. Studies on inter relationship between bulb yield and important plant character of onion, Veg. Sci., 12(1): 710.

Saimbhi, M. S. S, Padda, D. H and Singh, G. 1971.Evaluation of onion variety for dehydration traits. J. Food Sci. and Technology 8(3):149-150

Sethi ,V, Anand J. C and Bhagchandani, P. M 1993. Quality screening of white onion cultivars for use in dehydration. Indian Food Packer. 27 (5): 3-8.

Singh, D.K., Singh, L. and Pandey, U.B. 2004. Nutritional and medicinal values of onion and garlic. NHRDF, News letter Vol. XXIV (2): 4-10

Singh, L., Singh, SP. and Mishra, PK. Evaluation of onion varieties at Karnal. Nat. Hort. Res. Develop. Foundation 
News Letter, VI (3): 3-4

Sidhu, AS., Singh, S. and Thakur, MR. Variability and correlation studies in onion. Indian J. Hort., 43: 260-264

Singh, R. K., Dubey, B. K., Bhonde, S. R. and Gupta, R. P. 2010. Estimates of genetic variability, heritability and correlation in red onion (Allium cepa L.) advance lines. Indian J. Agric. Sci., 80 (2): 160163.

Singh, R. K.,Bhonde, S. R. and Gupta, R. P. 2011. Studies on performance of onion (Allium cepa L.) hybrids for higher yield. Allium and Umbelliferae Improv. News Lett.20: 21- 26
Singh, R. K., Bhonde, S. R. and Gupta, R. P. 2010. Performance studies on onion promising lines for yield and quality. Green Farming. Int. J. Agric. Hort. Appl. Sci., 2(2):170-172

Singh, R. K,Dubey, B. K., Singh, S. K. and Bhonde, S. R. 2011. Selection of high yielding and good keeping quality variety in red onion. Prog. Hort., 43 (2): 243-247

Verma, L.R., Pandey, U.B., Bhonde, S.R. and Shrivastava, K.J. 1999. Quality evaluation of different onion varieties for dehydration. NHRDF News Letter XIX (2 \& 3): 1-6

\section{How to cite this article:}

Singh, R. K., S. K. Singh and Tailor, A. K. 2020. Studies on Performance of White Onion Advance Lines for Growth and Yield for Selection of Quality Variety. Int.J.Curr.Microbiol.App.Sci. 9(06): 3668-3675. doi: https://doi.org/10.20546/ijcmas.2020.906.432 